\title{
Hypopharynx reconstruction for primary hypopharyngeal carcinoma: a retrospective study and literature review
}

\author{
Yu Heng", Duo Zhang", Xiaoke Zhu", Liang Zhou, Ming Zhang, Kenan Li, Lei Tao \\ Department of Otolaryngology, Shanghai Key Clinical Disciplines of Otorhinolaryngology, Eye \& ENT Hospital, Fudan University, Shanghai, \\ China \\ Contributions: (I) Conception and design: All authors; (II) Administrative support: L Zhou, M Zhang, K Li, L Tao; (III) Provision of study materials \\ or patients: Y Heng, D Zhang, X Zhu; (IV) Collection and assembly of data: Y Heng, D Zhang, X Zhu; (V) Data analysis and interpretation: Y Heng, \\ D Zhang, X Zhu; (VI) Manuscript writing: All authors; (VII) Final approval of manuscript: All authors. \\ "These authors contributed equally to this work. \\ Correspondence to: Lei Tao, PhD; Kenan Li, PhD. ENT Institute and Department of Otorhinolaryngology, Shanghai Key Clinical Disciplines of \\ Otorhinolaryngology, Eye \& ENT Hospital, Fudan University, Shanghai 200031, China. Email: doctortaolei@163.com; leeconan_smile@163.com.
}

Background: The study aims to retrospectively evaluate disparate reconstructive choices for defects in patients with primary hypopharyngeal carcinoma undergoing tumor resection.

Methods: One hundred and twenty-five patients of primary hypopharyngeal carcinamo conducted with hypopharynx reconstruction for restoring the defects causing by tumor ablation from 2003 to 2016 at Eye \& ENT Hospital of Fudan University were reviewed. Most of them were treated with the following three techniques ( $\mathrm{n}=115)$ : gastric pull-up (GPU), the pedicled pectoralis major myocutaneous flap (PMMF) and the radial forearm free flap (RFFF). GPU was used for those with cervical esophagus invasion requiring esophageal resection in our institution. We focus on the postoperative complication morbidity, function of deglutition 1 month after hospital discharge and disease-free survival (DFS) outcomes.

Results: One month after operation, 56 of 65 (86.2\%) achieved solid or semisolid diet with normal swallowing function for GPU, 8 of 12 (66.7\%) for RFFF and 20 of 33 (60.6\%) for PMMF respectively. Postoperative complications occured in 15 (23.1\%), 8 (66.7\%), 16 (48.5\%) for GPU, RFFF and PMMF respectively, including flap/anastomotic necrsis, pharyngeal fistula, wound infection (both recipient and donor sites), pulmonary and cardiovascular complications.

Conclusions: GPU showed satisfactory outcomes for reconstruction of patients with hypopharyngeal carcinoma requiring esophageal resection. For patients without cervical esophagus invasion and underwent partial or total pharyngectomy, both PMMF and RFFF can afford good coverage and reliable functional and survival outcome as well as postoperative complication morbidity. Moreover, the only predictive factor for incidence of postoperative complication was extent of surgery, as total hypopharyngectomy was more likely to develop complications after surgery than partial hypopharyngectomy.

Keywords: Hypopharyngeal carcinoma; pharyngectomy; reconstruction; functional outcome; survival

Submitted Sep 17, 2020. Accepted for publication Mar 05, 2021.

doi: $10.21037 /$ tcr-20-2910

View this article at: https://dx.doi.org/10.21037/tcr-20-2910

\section{Introduction}

Hypopharyngeal carcinoma has been reported to be one of the most aggressive of the primary head and neck cancers, characterized by a 5 -year overall survival rate range of
$25 \%$ to $40 \%(1,2)$. This poor prognosis is attributed to the cancer often remaining asymptomatic for an extended period and thus having reached an advanced stage at the time of diagnosis. Although a trend of radiotherapy for 
hypopharyngeal lesions has arisen in recent years (3), surgical resection continues to be the standard therapeutic choice, including partial or total hypopharyngectomy \pm laryngectomy, according to the extension of the lesion and the involved subsites. For the sake of reducing postoperative recurrence and mortality, a safe and macroscopic surgical margin is needed for hypopharyngeal carcinoma resection, which could result in large and even circumferential defects after extensive cancer removal (4). Presently, reconstruction for those defects remains a challenge in clinical practice.

To meet the needs of short interval to successful oral alimentation and for low postoperative complication rates and tolerating postoperative radiation, many reconstruction techniques have been put into clinical use, mainly containing the myocutaneous pedicled flaps, such as the pectoralis major, latissimus dorsi, supraclavicular artery, and submental flaps (SBMFs); visceral transposition, such as gastric pull-up (GPU) and jejunal or colon autograft; and fasciocutaneous free flaps, such as the anterolateral thigh (ALT) and the radial forearm (5-7). The choice should consider the sizes of defects, the patient's preference, and the surgeon's experience and skill in general. The ultimate purpose is to restore the continuity of the digestive tract and swallowing function, fill surgical defects, protect major cervical vessels, and prevent severe complications.

Three methods of reconstruction were performed, including GPU, the pedicled pectoralis major myocutaneous flap (PMMF), and the radial forearm free flap (RFFF), in the Eye \& ENT Hospital of Fudan University's Department of Otolaryngology-Head and Neck Surgery from 2003 to 2016. Other reconstructive methods, including the supraclavicular artery flap (SPCAF), the SBMF, and the latissimus dorsi pedicled flap (LDPF), were also conducted in several patients. The present study aims to retrospectively analyze the functional and survival outcomes of different reconstruction techniques for patients with primary hypopharyngeal carcinoma treated in our institution and compare our data with the previous literature to summarize the management of hypopharynx reconstruction for future reference.

We present the following article in accordance with the STROBE reporting checklist (available at https://dx.doi. org/10.21037/tcr-20-2910).

\section{Methods}

\section{Patients and evaluation}

One hundred and twenty-five patients diagnosed with primary hypopharyngeal cancer were underwent reconstruction for defects caused by various kinds of cancer resections. Of these reconstructions, 115 implemented the three primary reconstruction techniques (GPU, PMMF, and RFFF), two patients underwent LDPF reconstructions, four patients underwent SPCAF reconstructions, and four patients underwent SBMF reconstructions. All operations were performed by the same medical team at the Eye \& ENT Hospital of Fudan University's Department of Otolaryngology-Head and Neck Surgery from 2003 to 2016.

Patients' records were reviewed, including the demographic data; the stage of primary tumor; the tumor's gross, postoperative adjuvant treatments and complications; the time of first feeding; the number of hospital days; and the rate of recurrence-free survival (RFS). Patients were staged in accordance with the International Union Against Cancer's (UICC, 2002) TNM classification. The function of deglutition 1 month after hospital discharge was regarded as the primary functional outcome and has been evaluated. All the clinical data were obtained from patients' medical records. The study was conducted in accordance with the Declaration of Helsinki (as revised in 2013). The study was approved by ethics board of the Eye \& ENT Hospital of Fudan University (No. KJ2008-01) and individual consent for this retrospective analysis was waived.

\section{Follow-up}

The follow-up data of our study was obtained through interviews in person with patients in the hospital or contact with the patients or their relatives by telephone, from the date of operation until March 2019 or the day of tumor recurrence. Tumor recurrence included local recurrence and distant metastasis.

\section{Statistical analysis}

SPSS 24.0 was used for the statistical analysis. Means, 
Table 1 Patient characteristics

\begin{tabular}{|c|c|}
\hline Characteristics & Value \\
\hline Age (years), mean \pm SD & $55.12 \pm 8.08$ \\
\hline Gender (male/female) & $120 / 5$ \\
\hline \multicolumn{2}{|l|}{ Tumor size $(\mathrm{cm}), \mathrm{n}(\%)$} \\
\hline$<6.0$ & $97(77.6)$ \\
\hline$\geq 6.0$ & $28(22.4)$ \\
\hline \multicolumn{2}{|l|}{ T stage, n (\%) } \\
\hline T1-T2 & $20(16.0)$ \\
\hline T3-T4 & $105(84.0)$ \\
\hline \multicolumn{2}{|l|}{ N stage, n (\%) } \\
\hline No & $28(22.4)$ \\
\hline $\mathrm{N} 1$ & $28(22.4)$ \\
\hline $\mathrm{N} 2 \mathrm{a}$ & $4(3.2)$ \\
\hline $\mathrm{N} 2 \mathrm{~b}$ & $47(37.6)$ \\
\hline N2c & $18(14.4)$ \\
\hline \multicolumn{2}{|l|}{ Preoperative therapy, n (\%) } \\
\hline No & $22(17.6)$ \\
\hline Yes & $103(82.4)$ \\
\hline \multicolumn{2}{|l|}{ ASA, n (\%) } \\
\hline 1 & $5(4.0)$ \\
\hline 2 & $64(51.2)$ \\
\hline 3 & $53(42.4)$ \\
\hline 4 & $3(2.4)$ \\
\hline \multicolumn{2}{|l|}{ Surgical method, n (\%) } \\
\hline $\mathrm{PH}$ & $6(4.8)$ \\
\hline $\mathrm{PL}+\mathrm{PH}$ & $16(12.8)$ \\
\hline $\mathrm{TL}+\mathrm{PH}$ & $20(16.0)$ \\
\hline $\mathrm{TL}+\mathrm{TH}$ & $29(23.2)$ \\
\hline $\mathrm{TL}+\mathrm{TH}+$ esophagectomy & $54(43.2)$ \\
\hline \multicolumn{2}{|c|}{ Postoperative adjuvant treatment, n (\%) } \\
\hline No & $58(46.4)$ \\
\hline Yes & $67(53.6)$ \\
\hline
\end{tabular}

proportions, and standard deviations were calculated from the collected data. Comparisons of the data between two independent groups were performed using the Chi-square test and the independent $t$-test for corresponding variables. $\mathrm{P}<0.05$ was considered statistically significant. We also calculated the RFS using the Kaplan-Meier method and tested it through the log-rank test method.

\section{Results}

\section{Characteristics of patients}

One hundred and twenty-five patients diagnosed with primary hypopharyngeal carcinoma were included in the present study. All the patients received preoperative workups, including a physical examination, blood and urine examinations, a laryngeal endoscope, a computed tomography (CT) of the neck and chest, and a cardiological examination. The diagnoses were conformed histologically, and the postoperative histopathology of the patients in our series was all squamous cell carcinoma. The mean age was 55.1 years (ranging from 38 to 76 years old). Men accounted for the majority (male-to-female ratio of 120:5). All the patients were staged before operation according to the International Union Against Cancer's (UICC, 2002) TNM classification. The patients' tumors were staged T1 $(\mathrm{n}=2)$, T2 (n=18), T3 (n=79), T4 (n=26), N0 ( $=28)$, N1 ( $=28)$, N2a $(n=4), N 2 b(n=47), N 2 c(n=18)$. Twenty-two patients $(17.6 \%)$ underwent preoperative (chemo)radiotherapy, and 67 patients $(53.6 \%)$ received postoperative adjuvant therapy. See further details in Table 1 .

\section{Types of reconstruction}

Six different ways of reconstructing the surgical defects were included in our study, including three main techniques: GPU, PMMF, and RFFF. GPU was used for tumors with cervical esophagus invasions requiring esophageal resections, while PMMFs and RFFFs were conducted for tumors restricted to the hypopharynx and larynx and requiring only partial or total hypopharyngectomy \pm laryngectomy. Of the 125 patients, $65(52.0 \%)$ patients underwent partial or total pharyngolaryngectomy and esophagectomy with reconstruction by GPU, 38 (30.4\%) 
Table 2 Type of reconstruction

\begin{tabular}{lcc}
\hline Techniques & $\mathrm{N}$ & $\%$ \\
\hline RFFF & 12 & 9.6 \\
GPU & 65 & 52.0 \\
PMMF & 38 & 30.4 \\
SPCAF & 4 & 3.2 \\
SBMF & 4 & 3.2 \\
LDPF & 2 & 1.6 \\
\hline
\end{tabular}

RFFF, radial forearm free flap; GPU, gastric pull-up; PMMF, pedicled pectoralis major myocutaneous flap; SPCAF, supraclavicular artery flap; SBMF, submental flap; LDPF, latissimus dorsi pedicled flap.

patients underwent partial or total pharyngolaryngectomy with reconstruction by PMMF, and 12 (9.6\%) patients underwent partial or total pharyngolaryngectomy with reconstruction by RFFF. The other 10 (8.0\%) patients were reconstructed by LDPF, SBMF, and SPCAF. Details are shown in Table 2.

\section{Perioperative mortality and morbidity}

For the three main reconstruction techniques-GPU, PMMF, and RFFF_only one patient died because of cardiac and respiratory failure within 1 month postoperatively. The total incidence of postoperative complications in our study was $37.4 \%(n=43)$, including flap/ anastomotic necrosis, pharyngeal fistulas, incision infections (both recipient and donor sites), wound dehiscence, and pulmonary and cardiovascular complications, including $18(27.7 \%)$ patients in the GPU group, 17 (44.7\%) patients in the PMMF group, and $8(66.7 \%)$ patients in the RFFF group. Among these, a pharyngeal fistula was the most common postoperative complication, and 18 patients suffered from varying degrees of it, including 5 from the GPU group, 10 from the PMMF group, and 3 from the RFFF group. Most of those patients were cured with dressing changes and anti-infection therapy; only one patient in the GPU group and another patient in the RFFF group required another flap reconstruction using PMMF for salvage. Eight patients suffered from incision infections of the recipient or donor site or both, including 2 (3.1\%) in the GPU group, 5 (13.2\%) in the PMMF group, and $1(8.3 \%)$ in the RFFF group. Necrosis of the flap or anastomotic stoma occurred in $1(1.5 \%)$ patient in the GPU group, 1 (2.6\%) patient in the PMMF group, and 1 (16.7\%) patient in the RFFF group. Those cases were all repaired with PMMFs in second operations.

Other postoperative complications that needed surgical intervention were wound dehiscence (in three patients) and anastomotic stenosis (in two patients). Seven patients had pulmonary complications involving pulmonary edema, and infection and cardiac complications occurred in seven patients, including atrial fibrillation and tachycardia. Among them, patients in the GPU group accounted for the majority (see Table 3 for details). We also did a multivariable ordinal regression for postoperative complications, and the results showed no significant difference among the GPU, PMMF, and RFFF groups $(\mathrm{P}>0.05)$. The surgical method was found to be a significant predictor for postoperative complications, as total hypopharyngectomy was more likely to result in complications after surgery than partial hypopharyngectomy ( $\mathrm{P}=0.030$, as shown in Table 4).

For the other three kinds of reconstruction-LDPF, SBMP, and SPCAF - the incidences of postoperative complications were $50 \%(1 / 2), 25 \%(1 / 4)$, and $25 \%$ (1/4), respectively. The three patients with postoperative complications all suffered from pharyngeal fistulas and were cured after dressing changes and anti-infection therapy.

\section{Length of hospital stay}

The median and mean lengths of hospital stay in our study was 22.0 and 26.6 days (a range of 15-86 days): 23.0 and 27.3 days for the GPU group (15-86 days), 20.0 and 22.5 days for the PMMF group (15-36 days), and 25.5 and 30.4 days for RFFF (16-51 days) (see Table 5). The average length of hospital stay for patients in the PMMF group was shorter than that of patients in the GPU and RFFF groups, but no significant differences were found among the three groups (GPU group vs. PMMF group, $\mathrm{P}=0.093$; RFFF group vs. $\mathrm{PMMF}$ group, $\mathrm{P}=0.059$; and $\mathrm{RFFF}$ group $v$. GPU group, $\mathrm{P}=0.401$ ).

For the two patients who underwent LDPF reconstructions, the lengths of hospital stay were 22 and 23 days, respectively. As for SBMF and SPCAF, the average lengths of hospital stay were 23.0 and 25.5 days, respectively.

\section{Swallowing function}

For the 115 patients who underwent reconstruction utilizing one of the three main techniques, 100 patients 
Table 3 Postoperative complications of three main techniques

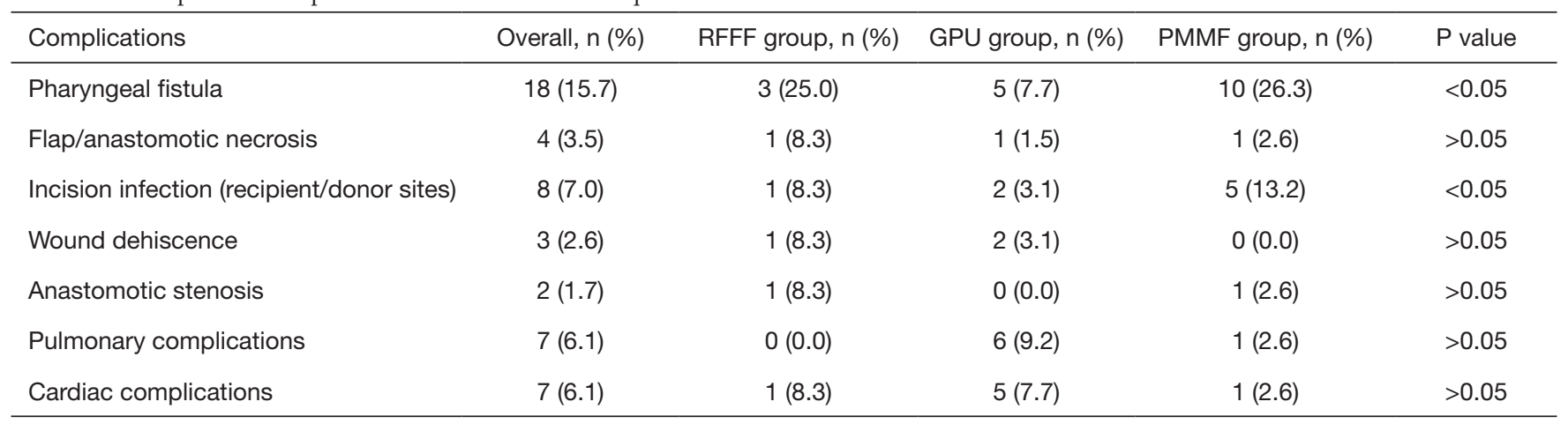

RFFF, radial forearm free flap; GPU, gastric pull-up; PMMF, pedicled pectoralis major myocutaneous flap.

Table 4 Multivariable ordinal regression for postoperative complications

\begin{tabular}{|c|c|c|}
\hline Variables & Odds ratio (95\% confidence interval) & $P$ value \\
\hline RFFF & $1.991(0.458-8.659)$ & 0.359 \\
\hline GPU & $1.320(0.306-5.692)$ & 0.709 \\
\hline PMMF & 1 & \\
\hline No & $1.274(0.442-3.671)$ & 0.654 \\
\hline Yes & 1 & - \\
\hline \multicolumn{3}{|l|}{ Postoperative adjuvant treatment } \\
\hline No & $1.606(0.682-3.778)$ & 0.278 \\
\hline \multicolumn{3}{|l|}{ Surgical methods } \\
\hline Partial hypopharyngectomy & $5.400(1.182-24.675)$ & 0.030 \\
\hline Total hypopharyngectomy & 1 & - \\
\hline
\end{tabular}

RFFF, radial forearm free flap; GPU, gastric pull-up; PMMF, pedicled pectoralis major myocutaneous flap.

Table 5 Hospital stay and resumption of oral feeding

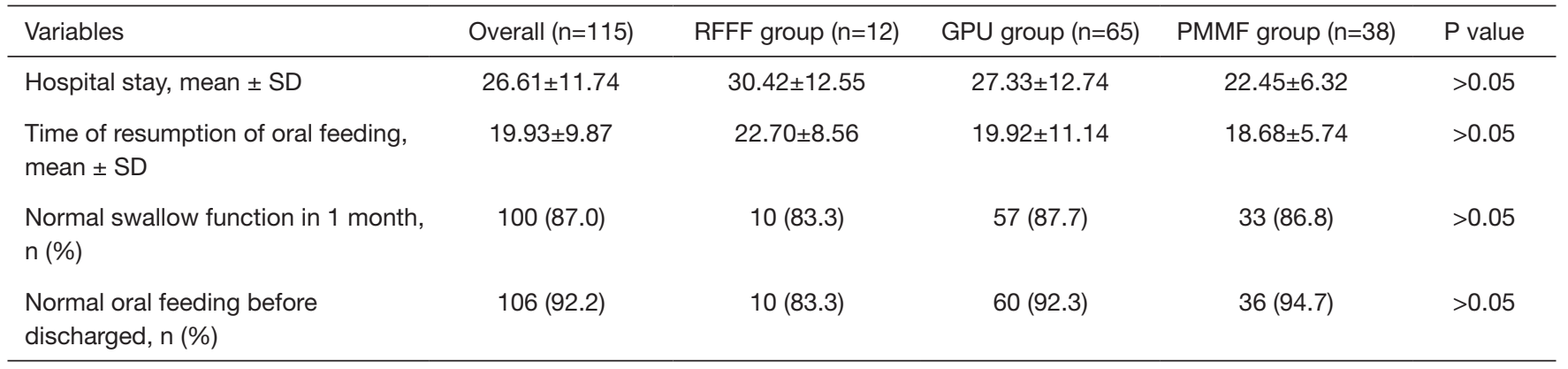

RFFF, radial forearm free flap; GPU, gastric pull-up; PMMF, pedicled pectoralis major myocutaneous flap. 


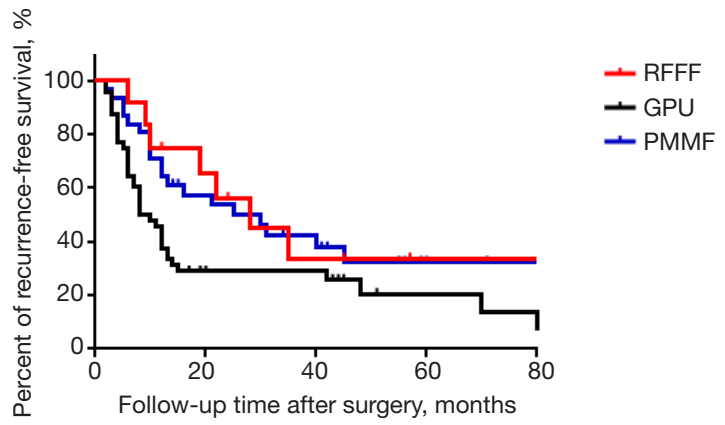

Figure 1 The RFS rates by different reconstruction techniques. RFS, recurrence-free survival; RFFF, radial forearm free flap; GPU, gastric pull-up; PMMF, pedicled pectoralis major myocutaneous flap.

(87.0\%) achieved a fluid or semifluid diet with normal swallow function within 1 month postoperatively: 57 of $65(87.7 \%)$ for the GPU group, 33 of 38 (86.8\%) for the PMMF group, and 10 of 12 (83.3\%) for the RFFF group, respectively. The median and mean time for the resumption of oral feeding after surgery was 17.0 days and 19.9 days (a range of 9-80 days): 16.0 and 19.2 days for the GPU group (range 9-80 days), 18.0 and 18.7 days for the PMMF group (a range of 11-32 days), and 21.5 and 22.7 days for the RFFF group (a range of 14-40 days) (see Table 5). The results showed no significant differences in the average time for the resumption of oral feeding between the three main techniques (GPU group vs. $\mathrm{PMMF}$ group, $\mathrm{P}=0.615$; RFFF group vs. GPU group, $\mathrm{P}=0.412$; and $\mathrm{RFFF}$ group vs. $\mathrm{PMMF}$ group, $\mathrm{P}=0.291$ ). One hundred and six patients achieved normal oral feeding after recovering from the operation and before being discharged. Among the other 9 patients, 3 patients required a nasal feeding tube and 2 could not eat a solid diet in the GPU group; 1 patient needed gastrostomy for a pharyngeal fistula and another patient could only consume fluids in the PMMF group; and for the RFFF group, 2 patients could take only fluid food when discharged.

Two patients in the LDPF reconstruction group and the 4 patients in the SPCAF group all achieved a fluid or semifluid diet within 1 month after surgery and achieved normal oral feeding when discharged. Only 1 of the 4 patients who received SBMF reconstruction underwent gastrostomy.

\section{Tumor recurrence}

Our average follow-up time was 23.4 months (a range of
1-120 months). The RFS rates by reconstruction technique are shown in Figure 1. The actuarial 1- and 3-year RFS rates were $37.5 \%$ and $28.9 \%$ for the GPU group, $64.5 \%$ and $42.1 \%$ for the PMMF group, and $75.0 \%$ and $33.8 \%$ for the RFFF group, respectively. The results showed no significant difference in the RFS rates between RFFF and PMMF groups $(\mathrm{P}=0.809)$, and patients in both the RFFF and PMMF groups had better RFS outcomes than the patients in the GPU group ( $\mathrm{P}=0.083$ and 0.039 , respectively).

\section{Discussion}

Primary hypopharyngeal carcinoma is a relatively rare disease with an unfavorable prognosis. Surgery is still the keystone of curative treatment. Head and neck surgeons choose different surgical procedures for different patients according to the extension of the tumor and the subsites involved. In some patients, the surgical defect can be primarily sutured, and oral feeding may be resumed after recovering from the surgery. However, some patients with extensive resections need flap reconstruction to repair the pharyngeal defect, preserve normal swallowing function, and restore the natural pharyngeal anatomic structure.

Various methods of flap reconstruction can be selected to repair the defects caused by the extensive resection of hypopharyngeal carcinoma. Depending on the size and the position of the defect, surgeons should select the most suitable one for their patients. Remarkably, all of the techniques have the ability to reintegrate the continuity of the upper digestive tract, and each technique has different rates of pharyngeal fistulas, graft necrosis, and successful oral feeding. No consensus has been reached on the optimal reconstruction methods for the large and even circumferential defects after hypopharyngectomy. Here we summarized reports containing the hypopharyngeal reconstruction options most frequently used, including myocutaneous pedicled flaps, visceral transposition flaps, and fasciocutaneous free flaps, shown respectively in Tables 6-8.

GPU has long been considered as the standard method of reconstruction after hypoharyngectomy beyond the thoracic inlet (8), and it remains the optimal choice for defects requiring the reconstruction of cervical esophagus in our institution. GPU has many advantages compared to the other techniques of reconstruction. First, there is only one anastomosis to be dealt with, which results in relatively low occurrence rates of pharyngeal fistulas and anastomotic stenosis, with rates reported in the last decade ranging 


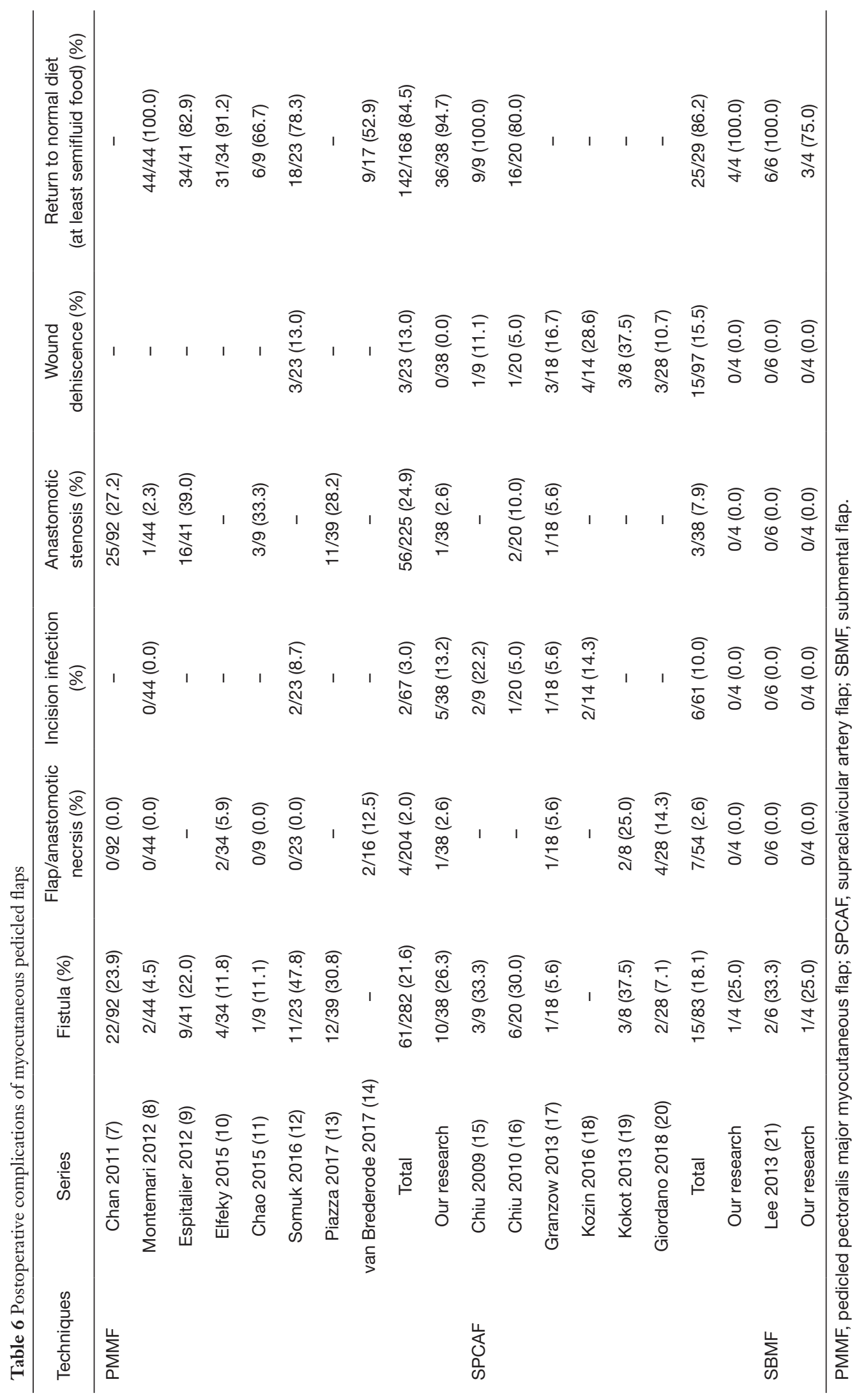


Table 7 Postoperative complications of visceral transposition

\begin{tabular}{|c|c|c|c|c|c|c|c|}
\hline Techniques & Series & Fistula (\%) & $\begin{array}{l}\text { Flap/ } \\
\text { anastomotic } \\
\text { necrsis (\%) }\end{array}$ & $\begin{array}{l}\text { Incision } \\
\text { infection (\%) }\end{array}$ & $\begin{array}{l}\text { Anastomotic } \\
\text { stenosis (\%) }\end{array}$ & $\begin{array}{l}\text { Wound } \\
\text { dehiscence (\%) }\end{array}$ & $\begin{array}{l}\text { Return to normal } \\
\text { diet (at least } \\
\text { semifluid food) (\%) }\end{array}$ \\
\hline \multirow{6}{*}{ GPU } & Fujioka 2017 (23) & 4/29 (13.8) & - & - & 6/29 (20.7) & - & - \\
\hline & He 2011 (24) & 19/208 (9.1) & - & 8/208 (3.9) & 7/208 (3.4) & - & - \\
\hline & Denewer 2014 (26) & $5 / 34(14.7)$ & - & - & 3/34 (8.8) & - & 28/34 (82.4) \\
\hline & Li 2018 (27) & $3 / 42(7.1)$ & $3 / 42(7.1)$ & 0/42 (0.0) & - & $2 / 42(4.8)$ & $39 / 42$ (92.9) \\
\hline & Elfeky 2015 (10) & $3 / 33(9.1)$ & $0 / 33(0.0)$ & - & - & - & $31 / 33$ (93.9) \\
\hline & Total & $47 / 453(10.4)$ & $3 / 75(4.0)$ & 11/357 (3.1) & 19/361 (5.3) & $2 / 42(4.8)$ & 98/109 (89.9) \\
\hline \multirow{6}{*}{ JFF } & Miyamoto 2011 (29) & $3 / 43(7.0)$ & $2 / 43(4.7)$ & $3 / 43(7.0)$ & $1 / 43(2.3)$ & - & 41/43 (95.3) \\
\hline & Moradi 2010 (30) & $2 / 43(4.7)$ & - & - & 6/43 (14.0) & - & - \\
\hline & Chan 2011 (7) & $4 / 86(4.7)$ & - & - & 2/86 (2.3) & - & - \\
\hline & Elfeky 2015 (10) & $3 / 37(8.1)$ & 2/37 (5.4) & - & - & - & $36 / 37(97.3)$ \\
\hline & Denewer 2014 (26) & 2/25 (8.0) & - & - & $1 / 25(4.0)$ & 3/28 (10.7) & 20/25 (80.0) \\
\hline & Total & 17/337 (5.0) & $5 / 183(2.7)$ & $3 / 43(7.0)$ & 10/197 (5.1) & 5/131 (3.8) & 200/208 (96.2) \\
\hline
\end{tabular}

GPU, gastric pull-up; JFF, jejunal free flap.

Table 8 Postoperative complications of fasciocutaneous free flaps

\begin{tabular}{|c|c|c|c|c|c|c|c|}
\hline Techniques & Series & Fistula (\%) & $\begin{array}{l}\text { Flap/ } \\
\text { anastomotic } \\
\text { necrsis (\%) }\end{array}$ & $\begin{array}{l}\text { Incision } \\
\text { infection (\%) }\end{array}$ & $\begin{array}{l}\text { Anastomotic } \\
\text { stenosis (\%) }\end{array}$ & $\begin{array}{l}\text { Wound } \\
\text { dehiscence (\%) }\end{array}$ & $\begin{array}{l}\text { Return to normal } \\
\text { diet (at least } \\
\text { semifluid food) (\%) }\end{array}$ \\
\hline \multirow{3}{*}{ RFFF } & van Brederode 2017 (14) & - & 4/31 (12.9) & - & - & - & $20 / 25(80.0)$ \\
\hline & Piazza 2017 (13) & $5 / 46(10.9)$ & $1 / 46(2.2)$ & - & $2 / 46(4.3)$ & - & - \\
\hline & Our research & $3 / 12(25.0)$ & $2 / 12(16.7)$ & 1/12 (8.3) & 1/12 (8.3) & 2/65 (3.1) & 10/12 (83.3) \\
\hline \multirow[t]{4}{*}{ ALT } & van Brederode 2017 (14) & - & $1 / 11(9.1)$ & - & - & - & $6 / 9(66.7)$ \\
\hline & Piazza 2017 (13) & 2/59 (3.4) & $0 / 4(0.0)$ & - & $1 / 59(1.7)$ & - & - \\
\hline & Huang 2015 (35) & 8/45 (17.8) & - & - & $4 / 45(8.9)$ & - & $34 / 45(75.6)$ \\
\hline & Total & 23/169 (13.6) & $5 / 86(5.8)$ & - & $10 / 169(5.9)$ & $1 / 61(1.6)$ & $90 / 123(73.2)$ \\
\hline
\end{tabular}

RFFF, radial forearm free flap; ALT, anterolateral thigh. 
from $5.9-14.7 \%(10,22-27)$ and $3.3-20.7 \%(22-24,26)$, respectively. The overall incidences that were summarized occurred in $10.4 \%$ and $5.3 \%$ of patients, respectively (shown in Table 7). In this study, among the 65 patients treated with GPU, only 5 (7.7\%) suffered from fistula formation and none $(0.0 \%)$ had postoperative anastomotic stenosis. The low rate of fistula formation and stricture was verified. Other postoperative complications, including flap/ anastomotic necrosis $(1.5 \%)$, incision infection $(3.1 \%)$, and wound dehiscence $(4.8 \%)$, occurred in a small minority of patients, both in our study and in research over the last decade (see Table 7).

Another advantage of GPU is the rapid rehabilitation of swallowing and generally positive functional outcomes. The reported rate of returning to a semifluid or solid food diet ranges from $82.4 \%$ to $93.9 \%(10,26,27)$, and in our study, 57 of $65(87.7 \%)$ patients achieved oral intake within 1 month and $60(92.3 \%)$ patients achieved normal oral feeding before being discharged, which suggests that this reconstruction technique is a reliable method with a satisfactory swallowing outcome. Additionally, because of the excellent blood supply of the stomach, the rate of necrosis is extremely low ( $4.0 \%$ reported and $1.5 \%$ in this study). However, as Elfeky (10) reported, pulmonary complications are more common after GPU than other techniques of reconstruction. Our data showed that pulmonary and cardiac complications occurred in $11(16.9 \%)$ patients after GPU that only emerged in $1(8.3 \%)$ and $2(5.2 \%)$ patients after RFFF and PMMF, respectively. This suggests that patients undergoing GPU have an increased susceptibility to pulmonary-cardiac complications.

As for RFS outcomes, the GPU group showed a relatively lower RFS rate than the PMMF $(\mathrm{P}=0.038)$ and $\mathrm{RFFF}$ $(\mathrm{P}=0.083)$ groups in our series. We believe that, primarily, this is because most of the patients who underwent GPU in our series had tumor invasions of the esophagus needing esophagectomy that were regarded as advanced.

In Clark's report, PMMF and fasciocutaneous free flaps were used most in the reconstruction of partial pharyngeal defects (36). In our institution, for patients receiving pharyngolaryngectomy without esophageal resection whose defects were above the thoracic inlet, myocutaneous pedicled flaps (mainly involving PMMF) and fasciocutaneous free flaps (using RFFF) were performed. As we know, PMMF is easy to harvest and rich in blood supply. Meanwhile, no requirement for microvascular reconstruction makes it widely applied in the filling of defects after hypopharyngectomy, even in less experienced centers and for patients in poor condition (e.g., aged and emaciated). However, with the improvement of microsurgical techniques, PMMF has gradually been replaced by free flaps in some institutions. Two main kinds of fasciocutaneous free flaps-RFFF and anterolateral thigh (ALT) flaps-are thin, soft, and characterized by their reliability, good vascularity, ease of acquisition, and low donor site morbidity $(6,37)$. In our study, a comparison was conducted between PMMF and RFFF.

The occurrence of a pharyngeal fistula is the most common and menacing postoperative complication in both PMMF and RFFF groups, with incidence rates of $26.3 \%$ $(\mathrm{n}=10)$ and $25.0 \%(\mathrm{n}=3)$, respectively. Most patients with a less severe fistula were cured with dressing changes and antibiotic therapy, except for one patient in the RFFF group who required another flap reconstruction (using PMMF) for salvage. Flap necrosis is another serious complication in hypopharyngeal reconstruction. The reported necrosis rate was slightly higher in the RFFF group than in the PMMF group (6.5\% vs. $2.0 \%$, shown in Tables 6,8 ). In our study, both RFFF (8.3\%) and PMMF (2.6\%) had low occurrence rates of flap or anastomotic necrosis, in line with previous studies. Other postoperative complications, including incision infection, wound dehiscence, and anastomotic stenosis, occurred infrequently in both the PMMF and RFFF groups, and all could be cured with conventional methods. Multivariable ordinal regression also exhibited no significant difference among GPU, PMMF, and RFFF groups in postoperative complications.

As for length of hospital stay and swallowing rehabilitation within 1 month, the RFFF group showed comparable outcomes with the PMMF group $(\mathrm{P}>0.05)$. All 12 patients in the RFFF group regained oral feeding when discharged, but two of them could only consume fluids. These two groups also had similar RFS outcomes $(\mathrm{P}=0.809)$, demonstrating that both techniques appear to be reliable options for the reconstruction of defects above the thoracic inlet after hypopharyngectomy.

Although not often used, other options, including LDPF, SBMF, and SPCAF, showed positive functional outcomes in our institution and again proved that myocutaneous pedicled flaps are effective methods of hypopharyngeal reconstruction.

\section{Conclusions}

GPU showed satisfactory outcomes for the reconstruction of patients with hypopharyngeal carcinoma requiring 
esophageal resection. For patients without cervical esophagus invasions who underwent partial or total pharyngectomy, both PMMF and RFFF offered good coverage and reliable functional and survival outcomes as well as limited postoperative complications.

\section{Acknowledgments}

Funding: This study was supported by the Science and Technology Innovation Project of Shanghai ShenKang Hospital Clinical Development Center under Grand (SHDC12015114); the Science and Technology Commission of Shanghai Municipality under Grand (16411950100); the National Natural Science Foundation of China under Grand (81772878, 30801283, 30972691); the Shanghai Science and Technology Development Funds under Grand (09QA1401000, 10QA1405900, 14411961900); the Training Program of the Excellent Young Talents of Shanghai Municipal Health System under Grand (XYQ2011055, XYQ2011015); and the Shanghai Municipal Science and Technology Foundation under Grand (11JC1410802).

\section{Footnote}

Reporting Checklist: The authors have completed the STROBE reporting checklist. Available at https://dx.doi. org/10.21037/tcr-20-2910

Data Sharing Statement: Available at https://dx.doi. org/10.21037/tcr-20-2910

Conflicts of Interest: All authors have completed the ICMJE uniform disclosure form (available at https://dx.doi. org/10.21037/tcr-20-2910). The authors have no conflicts of interest to declare.

Ethical Statement: The authors are accountable for all aspects of the work in ensuring that questions related to the accuracy or integrity of any part of the work are appropriately investigated and resolved. The study was conducted in accordance with the Declaration of Helsinki (as revised in 2013). The study was approved by ethics board of the Eye \& ENT Hospital of Fudan University (No. $\mathrm{KJ} 2008-01)$ and individual consent for this retrospective analysis was waived.

Open Access Statement: This is an Open Access article distributed in accordance with the Creative Commons Attribution-NonCommercial-NoDerivs 4.0 International License (CC BY-NC-ND 4.0), which permits the noncommercial replication and distribution of the article with the strict proviso that no changes or edits are made and the original work is properly cited (including links to both the formal publication through the relevant DOI and the license). See: https://creativecommons.org/licenses/by-nc-nd/4.0/.

\section{References}

1. Peracchia A, Bonavina L, Botturi M, et al. Current status of surgery for carcinoma of the hypopharynx and cervical esophagus. Dis Esophagus 2001;14:95-7.

2. Wycliffe ND, Grover RS, Kim PD, et al. Hypopharyngeal cancer. Top Magn Reson Imaging 2007;18:243-58.

3. Newman JR, Connolly TM, Illing EA, et al. Survival trends in hypopharyngeal cancer: a population-based review. Laryngoscope 2015;125:624-9.

4. van der Putten L, Spasiano R, de Bree R, et al. Flap reconstruction of the hypopharynx: a defect orientated approach. Acta Otorhinolaryngol Ital 2012;32:288-96.

5. Welkoborsky HJ, Deichmüller C, Bauer L, et al. Reconstruction of large pharyngeal defects with microvascular free flaps and myocutaneous pedicled flaps. Curr Opin Otolaryngol Head Neck Surg 2013;21:318-27.

6. Piazza C, Taglietti V, Nicolai P. Reconstructive options after total laryngectomy with subtotal or circumferential hypopharyngectomy and cervical esophagectomy. Curr Opin Otolaryngol Head Neck Surg 2012;20:77-88.

7. Chan YW, Ng RW, Liu LH, et al. Reconstruction of circumferential pharyngeal defects after tumour resection: reference or preference. J Plast Reconstr Aesthet Surg 2011;64:1022-8.

8. Montemari G, Rocco A, Galla S, et al. Hypopharynx reconstruction with pectoralis major myofascial flap: our experience in 45 cases. Acta Otorhinolaryngol Ital 2012;32:93-7.

9. Espitalier F, Ferron C, Leux C, et al. Results after U-shaped pectoralis major myocutaneous flap reconstruction of circumferential pharyngeal defects. Laryngoscope 2012;122:2677-82.

10. Elfeky AE, Nasr WF, Khazbak A, et al. Hypopharyngeal reconstruction: a comparison of three alternatives. Eur Arch Otorhinolaryngol 2015;272:3045-50.

11. Chao JW, Spector JA, Taylor EM, et al. Pectoralis major myocutaneous flap versus free fasciocutaneous flap for reconstruction of partial hypopharyngeal defects: what should 
we be doing? J Reconstr Microsurg 2015;31:198-204.

12. Somuk BT, Çiftçi O, Aygenç E. Evaluation of cases who underwent reconstruction through pectoralis major myocutaneous flap after laryngopharyngectomy. Turk Arch Otorhinolaryngol 2016;54:58-62.

13. Piazza C, Bon FD, Paderno A, et al. Fasciocutaneous free flaps for reconstruction of hypopharyngeal defects. Laryngoscope 2017;127:2731-7.

14. van Brederode TD, Halmos GB, Stenekes MW. Functional outcome after one-stage flap reconstruction of the hypopharynx following tumor ablation. Eur Arch Otorhinolaryngol 2017;274:969-76.

15. Chiu ES, Liu PH, Friedlander PL. Supraclavicular artery island flap for head and neck oncologic reconstruction: indications, complications, and outcomes. Plast Reconstr Surg 2009;124:115-23.

16. Chiu ES, Liu PH, Baratelli R, et al. Circumferential pharyngoesophageal reconstruction with a supraclavicular artery island flap. Plast Reconstr Surg 2010;125:161-6.

17. Granzow JW, Suliman A, Roostaeian J, et al. Supraclavicular artery island flap (SCAIF) vs free fasciocutaneous flaps for head and neck reconstruction. Otolaryngol Head Neck Surg 2013;148:941-8.

18. Kozin ED, Sethi RK, Herr M, et al. Comparison of perioperative outcomes between the supraclavicular artery island flap and fasciocutaneous free flap. Otolaryngol Head Neck Surg 2016;154:66-72.

19. Kokot N, Mazhar K, Reder LS, et al. The supraclavicular artery island flap in head and neck reconstruction: applications and limitations. JAMA Otolaryngol Head Neck Surg 2013;139:1247-55.

20. Giordano L, Di Santo D, Bondi S, et al. The supraclavicular artery island flap (SCAIF) in head and neck reconstruction: an Italian multi-institutional experience. Acta Otorhinolaryngol Ital 2018;38:497-503.

21. Lee JC, Chu YH, Lin YS, et al. Reconstruction of hypopharyngeal defects with submental flap after laryngopharyngectomy. Eur Arch Otorhinolaryngol 2013;270:319-23.

22. An CM, Wang SX, Lyu F, et al. Gastric pull-up reconstruction after pharyngoesphagectomy for advanced hypopharyngeal or cervical esophageal squamous cell carcinoma. Zhonghua Er Bi Yan Hou Tou Jing Wai Ke Za Zhi 2016;51:740-5.

23. Fujioka M, Hayashida K, Fukui K, et al. Venous superdrained gastric tube pull-up procedure for hypopharyngeal and cervical esophageal reconstruction reduces postoperative anastomotic leakage and stricture.
Dis Esophagus 2017;30:1-6.

24. He S, Sun J, Wang Y, et al. Complication following gastric pull-up reconstruction for advanced hypopharyngeal or cervical esophageal carcinoma: a 20-year review in a Chinese institute. Am J Otolaryngol 2011;32:275-8.

25. Sreehariprasad AV, Krishnappa R, Chikaraddi BS, et al. Gastric pull up reconstruction after pharyngo laryngo esophagectomy for advanced hypopharyngeal cancer. Indian J Surg Oncol 2012;3:4-7.

26. Denewer A, Khater A, Hafez MT, et al. Pharyngoesophageal reconstruction after resection of hypopharyngeal carcinoma: a new algorithm after analysis of 142 cases. World J Surg Oncol 2014;12:182.

27. Li SC, Li ZD, Liu HW, et al. Application of gastric pull up and complex laryngotracheal flap to reconstruct the circumferencial defect after resection of the hypopharyngeal and cervical esophageal cancers. Zhonghua Er Bi Yan Hou Tou Jing Wai Ke Za Zhi 2018;53:661-7.

28. Xu W, Lyu ZH, Zou JD, et al. Reconstruction with free jejuna flap for the defect after removal of hypopharyngeal and cervical esophageal caneer: clinical analyses of 103 cases. Zhonghua Er Bi Yan Hou Tou Jing Wai Ke Za Zhi 2016;51:914-7.

29. Miyamoto S, Sakuraba M, Asano T, et al. Free jejunal patch graft for reconstruction after partial hypopharyngectomy with laryngeal preservation. Arch Otolaryngol Head Neck Surg 2011;137:181-6.

30. Moradi P, Glass GE, Atherton DD, et al. Reconstruction of pharyngolaryngectomy defects using the jejunal free flap: a 10-year experience from a single reconstructive center. Plast Reconstr Surg 2010;126:1960-6.

31. Joo YH, Sun DI, Park JO, et al. Factors predicting fistula following radial forearm free flap reconstruction for head and neck cancer. Oral Oncol 2010;46:684-7.

32. Spyropoulou GC, Lin PY, Chien CY, et al. Reconstruction of the hypopharynx with the anterolateral thigh flap: defect classification, method, tips, and outcomes. Plast Reconstr Surg 2011;127:161-72.

33. Bianchi B, Ferri A, Ferrari S, et al. The free anterolateral thigh musculocutaneous flap for head and neck reconstruction: one surgeon's experience in 92 cases. Microsurgery 2012;32:87-95.

34. Zelken JA, Kang CJ, Huang SF, et al. Refinements in flap design and inset for pharyngoesophageal reconstruction with free thigh flaps. Microsurgery 2017;37:112-8.

35. Huang TC, Hsu YC, Chen HC, et al. Functional outcome analysis after anterolateral thigh flap 
reconstruction of pharyngoesophageal defect. Ann Plast Surg 2015;75:174-9.

36. Clark JR, Gilbert R, Irish J, et al. Morbidity after flap reconstruction of hypopharyngeal defects. Laryngoscope

Cite this article as: Heng $\mathrm{Y}$, Zhang D, Zhu X, Zhou L, Zhang M, Li K, Tao L. Hypopharynx reconstruction for primary hypopharyngeal carcinoma: a retrospective study and literature review. Transl Cancer Res 2021;10(7):3236-3247. doi: 10.21037/tcr20-2910
2006;116:173-81.

37. Ki SH, Choi JH, Sim SH. Reconstructive trends in postablation patients with esophagus and hypopharynx defect. Arch Craniofac Surg 2015;16:105-13. 